\title{
Guideline for the clinical use and dispensing of thalidomide*
}

\author{
R. J. POWELL \& J. M. M. GARDNER-MEDWIN \\ Clinical Immunology Unit, Immunology Department, Queen's Medical \\ Centre, University Hospital, Nottingham NG7 2UH, UK
}

Accepted 7 July 1994

\section{Introduction}

In the 1960s thalidomide virtually disappeared from clinical use after it was demonstrated that it is both a causative agent of severe irreversible peripheral neuropathy ${ }^{1,2}$ and a human teratogen. ${ }^{3,4}$ Currently in the UK there are no product licences for thalidomide but it can be prescribed on a 'named patient' basis in accordance with Section 9(1) of the Medicines Act $1968,{ }^{5}$ and its subsidiary legislation. ${ }^{6}$ It is being prescribed by hospital-based physicians to a small number of patients who have exhausted other therapeutic options. Hospital doctors who prescribe thalidomide should have the necessary expertise in its use and the resources to detect subclinical neuropathy. There is the potential for an increase in its use in conditions such as bone marrow transplantation ${ }^{7}$ and HIV-related disease. ${ }^{8}$ Even in these new areas, thalidomide should only become an option when all other therapeutic modalities have failed.

This continued, albeit limited, use of thalidomide has been criticized by some clinicians,, 10 and by individuals affected by thalidomide ${ }^{11}$ because of the known serious side effects of the drug. One of their concerns is that there are no legal restrictions or guidelines regulating its clinical use. Its current use is subject to the requirements of the laws governing the supply of a medicine for a 'named patient' prescription. ${ }^{5,6,12,13}$ This guideline is designed to promote the safest possible clinical use and dispensing of thalidomide.

These recommendations may require revision and modification as further clinical experience with thalidomide is gained. For that reason it is preferable that its clinical use should be regulated by guidelines rather than by law. However, it cannot be overstated that the risks of teratogenicity and peripheral neuropathy must be recognized, and addressed in each and every patient.

\section{(A) Clinical use}

1. Only severe disabling conditions that cause an unacceptable interference with normal life

* Reproduced with full permission of the BMJ Publishing Group from the Postgraduate Medical Journal (1994) 70: 901-904. 
should be treated with thalidomide, and only after other treatments have been tried and failed.

2. Pregnancy should be excluded before instituting therapy with thalidomide, specifically by a negative pregnancy test within 2 weeks prior to starting therapy.

3. Patients should be specifically excluded from treatment with thalidomide for any of the following reasons:

a. Unwilling to sign a consent form.

b. Unable to understand the potential risk from the use of thalidomide.

c. Unlikely to be able to comply with the prescribing instructions.

d. Women who wish to become pregnant.

e. Women of childbearing potential:

i. who have not practised a reliable form of contraception for 1 year;

ii. who are unwilling to take reliable contraceptive precautions;

iii. who are considered not capable of complying with the requirements for reliable contraception. Reliable contraceptive methods include the contraceptive pill, an intrauterine device, surgical sterilization of patient or sole partner. Female patients who do not normally practise contraception because of a history of infertility should do so whilst taking thalidomide.

4. Fully informed consent should be obtained using a written consent form and a signed agreement.

5. Women of childbearing potential should agree to stop taking thalidomide immediately should they miss a period, and urgently contact their prescribing physician. A pregnancy test should be provided and, if positive, appropriate counselling should be given.

6. Women of childbearing potential who discontinue treatment with thalidomide should agree to take reliable contraceptive precautions for 3 months after discontinuing thalidomide.

7. Patients should agree to return any unused supply of thalidomide to the prescribing physician.

\section{(B) Monitoring}

1. Appropriate clinical and electrophysiological measurements should be recorded before treatment is commenced. For certain conditions, photographs may be useful to monitor the progress of treatment.

2. The anticipated duration of treatment at which benefits of therapy will be judged should be agreed with the patient and treatment critically reviewed at the end of that period. Treatment failure must be recognized to avoid unnecessarily extended courses of thalidomide.

3. Follow-up visits should be at monthly intervals or less for the first 3 months to enable the clinician to detect side effects/early signs of toxicity. The warnings about the possible toxicity and the need for adequate contraception should be reinforced. Adequate time should be allowed to answer all questions raised by the patient.

4. All adverse events should be recorded and serious events notified to the Clinical Trials Section. Medicines Control Agency.*

* Clinical Trial Section, Medicines Control Agency, Room 1418 Market Towers, 1 Nine Elms Lane, London SW8 5NQ, UK. Tel. 0171-273 0327. 
5. Electrophysiological measurements (see below) should be repeated after each $10 \mathrm{~g}$ increment in total dose or 6 monthly, whichever is the sooner, for the duration of therapy.

6. Patients should be warned, and understand, that they must stop thalidomide immediately if paraesthesiae develop. In some cases the sensory loss may be permanent and adequate diagnosis, management and follow-up for these patients should be arranged.

\section{(C) Electrophysiological measurements}

1. Peripheral neuropathy is a common, severe and of ten irreversible side effect of treatment with thalidomide. Every effort must be made to detect this presymptomatically by electrophysiological techniques. Unfortunately there are no published electrophysiological studies that outline the criteria to predict the development of paraesthesiae. Should paraesthesiae develop, then thalidomide must be stopped immediately to limit further damage.

2. Electrophysiological testing should be performed at a constant temperature, by a consistent technique and by the same neurophysiologist, to provide at least one, preferably two, pretreatment baseline measurements of sensory nerve action potential amplitudes (SNAP). If more than one pretreatment value is available, confidence limits can be calculated for the individual patient.

3. The SNAP amplitudes should be measured in at least three nerves, for example, median, ${ }^{14}$ radial $^{15}$ and sural. ${ }^{16}$ A summated score with equal weighting for each nerve can be used to reduce the dominant contribution from the radial nerve SNAP amplitude. Nerve conduction velocities would not be expected to show significant changes in the early phase of an axonal neuropathy. ${ }^{17}$

4. Based on available data, a fall from the baseline summated score of $>40 \%$ should be regarded as significant. ${ }^{18}$

5. For those patients with a fall from baseline summated score of between $30 \%$ and $40 \%$, the intervals should be reduced between measurements and, therefore, the need to use thalidomide should be reviewed.

\section{(D) Patient information}

1. Each patient being treated with thalidomide should be given an information sheet (Figure 1).

2. A doctor prescribing thalidomide on a 'named patient' basis is entirely responsible for the patient's welfare. He must inform the patient of any contraindications, warnings and precautions associated with the use of the drug. To comply with the law, ${ }^{12}$ suppliers of a drug for a 'named patient' prescription must provide information about the drug on the containers and packages, but are not required to provide contraindications, warnings and precautions.

3. A sample patient information sheet is provided, which contains information relating to its proposed use and warnings about the potential, severe side effects of thalidomide. It should be updated as required. 


\section{(E) Manufacture and dispensing}

1. Thalidomide does not have a product licence in the UK. Nevertheless, a manufacturer or supplier may supply it to a medical practitioner for a prescription for a particular patient ${ }^{6}$ ('named patient' supply) provided that the manufacturer has a manufacturer's licence for 'specials'. 19

2. Staff and equipment at the manufacturing site should be adequate to ensure that the product is of the nature and quality specified by the doctor or pharmacist. Manufacture should be under proper supervision and adequately controlled.

3. Adequate records should be kept by the manufacturer/supplier. Records should include the amount of thalidomide that has been made, the form of the finished product, the 'named patient', the prescribing doctor and the person to whom it has been supplied.

4. The supplier should satisfy himself beyond doubt that orders are from hospital-based consultants who have knowledge of the use of thalidomide and its side effects.

5. It is recommended that the supplier should require that the order should be made in writing with the name of the patient, the prescribing doctor and the hospital address and telephone number. The letter should include a statement that the doctor is familiar with the use of thalidomide and its side effects, including peripheral neuropathy and teratogenicity. Also, a written assurance should be obtained that the drug will only be dispensed by the hospital pharmacist to the 'named patient' in accordance with the prescription.

6. Orders to provide a stock for a hospital pharmacy should not be accepted. However, an amount to provide for 3 months prescription for a 'named patient' could be supplied to be held in the pharmacy.

\section{(F) Labelling}

1. The labelling of containers and packages for medicines supplied for 'named patient' prescriptions are regulated by law. ${ }^{12}$

2. All particulars should be clear, legible and readily discernible so that they can be easily read. The particulars to be shown on the container should normally be shown on the body of the container.

3. Every container for thalidomide should be labelled to show the following information:

- The non-proprietary name or a proprietary designation. In addition the label should show a warning: 'Contains thalidomide'.

- The quantitative particulars in a conspicuous position. The labelling should distinguish between active and non-active ingredients.

- The quantity of thalidomide in the container or package.

- Any special requirements for the handling and storage, and the expiry date.

- The batch reference number, the number of the manufacturer's licence (preceded by $\mathrm{ML}$ ), and the name and address of the person who manufactured the product.

- The container should also show the warnings: 'Do not exceed the stated dose', 'Keep out of the reach of children', 'Thalidomide causes serious damage to babies if taken by women during pregnancy' and 'This drug must not be shared with anyone else.' 


\section{PATIENT INFORMATION SHEET FOR THALIDOMIDE USE}

in ...................................... (patient's name)

Thalidomide is a drug which can have severe side effects. This means it can only be used to treat a few debilitating conditions in which alternative treatments have been tried and failed. Thalidomide must be used with great care by patients and doctors and treatment will involve careful monitoring. Despite these drawbacks, in some patients thalidomide can be of significant benefit.

Condition being treated

How is the treatment given, how often and for how long?

$\operatorname{Dr}$ at Hospital

Tel. no. has prescribed thalidomide (proprietary name if used) for you.

The dose is $\mathrm{mg}=$ tablets and should be taken daily at night for . days.

\section{Hospital visits}

This treatment is monitored in the out-patients clinic, initially with monthly visits. You will be asked to have an electrical nerve test at regular intervals. These nerve tests can cause some discomfort but are an essential aspect of monitoring.

\section{Does the drug have side effects?}

1. Morning drowsiness is the most noticeable problem. This varies in each individual and may require your doctor to reduce the dose. Drowsiness may impair your ability to drive and operate machinery.

2. Nerve damage: Pins and needles of hands and feet are early signs of nerve damage and can develop after repeated courses or regular administration of thalidomide. Should you develop pins and needles you must stop thalidomide immediately and contact your hospital doctor. This is not uncommon and can be both severe and irreversible.

The aim of the electrical tests is to detect nerve damage before symptoms develop, and these will be a crucial part of your follow-up assessments. Should damage become apparent on the nerve test, thalidomide will be stopped, halting further deterioration in nerve function. Any damage at this stage would be so small it would be unnoticeable, but you would not be given thalidomide again.

3. Damage to babies: This is very important for all women considering thalidomide. Thalidomide is toxic to the developing baby, especially in the early months of pregnancy. If you wish to consider thalidomide you must be prepared to use adequate contraception throughout the duration of thalidomide therapy and for 3 months after it has finished. Should contraception fail, any resulting pregnancy may incur damage to the baby and consequently, if you miss a period at any time during treatment, you must stop thalidomide immediately and contact the doctor who prescribed the thalidomide. A pregnancy test would then be arranged and appropriate counselling given. Should pregnancy be confirmed, further investigations to assess any damage to the baby would be indicated. Your doctor can advise you about adequate contraception. No effects on male sperm are recognized.

4. Minor side effects such as constipation, nausea, dizziness, headaches and rarely skin rashes can occur.

\section{Having read this sheet}

This treatment involves you in possible risks and benefits. You should not agree to start thalidomide until you clearly understand these. Even if your doctor recommends the treatment you are free to ref use it and this will not in any way influence the rest of your care.

\section{Remember}

Thalidomide is a potentially dangerous medication. It must be securely stored away from children and only taken by the person to whom it is supplied.

Figure 1. Patient information sheet for thalidomide use. 


\section{References}

${ }^{1}$ Burley D. Is thalidomide to blame? $\operatorname{Br}$ Med J, 1961; 1: 130.

2 Fullerton PM, Kremer M. Neuropathy after intake of thalidomide (Distaval). Br Med J, 1961; 2: 853-858.

3 McBride WG. Thalidomide and congenital abnormalities (letter). Lancet, 1961; 2: 1358.

${ }^{4}$ Lenz W. Thalidomide and congenital abnormalities (letter). Lancet, 1962; 1: 45.

5 The Medicines Act 1968. HMSO, London, 1968.

6 The Medicines (Exemption from Licences) (Special Cases and Miscellaneous Provisions) Order 1972. HMSO, London, 1972.

7 Heney D, Norfolk DR, Wheeldon $\mathrm{J}$ et al. Thalidomide treatment for chronic graft versus-host disease. $\mathrm{Br} J$ Haematol, 1991; 78: 23-27.

8 Makonkawkeyoon S, Limson-Probre RN, Moreira AL, Schauf V \& Kaplan G. Thalidomide inhibits the replication of human immunodeficiency virus type 1. Proc Natl Acad Sci USA, 1993; 90: 5974-5978.

9 Crawford CL. Use of thalidomide in leprosy (letter). Br Med J, 1991; 302: 1603-1604.

${ }^{10}$ Hawkins DF. Thalidomide for systemic lupus erythematosus (letter). Lancet, 1992; 339: 1057.

11 Drug protest; thalidomide. The Sunday Times, 14 April 1991.

12 The Medicines (Labelling) Regulations (Regulations 11(1) (b) (i) and 11(1) (b) (ii) 1976). HMSO, London, 1976.

13 The Medicines (Exemption from Licences) (Importation) Order 1984. HMSO, London, 1984.

14 Gilliatt RW, Sears TA. Sensory action potentials in patients with peripheral nerve lesions. J Neurol Neurosurg Psych, 1958; 21: 109-118.

15 Downie AW, Scott TR. An improved technique for radial nerve conduction studies. J Neurol Neurosurg Psych, 1967; 30: 332-336.

16 Burke D, Skuse NF, Lethlean AK. Sensory conduction of the sural nerve in polyneuropathy. J Neurol Neurosurg Psych, 1974; 37: 647-652.

17 Fullerton PM, O’Sullivan DJ. Thalidomide neuropathy: a clinical electrophysiological and histological follow-up study. J Neurol Neurosurg Psych, 1968; 31: 543-551.

18 Gardner-Medwin JMM, Smith NJ, Powell RJ. Clinical experience with thalidomide in the management of severe oral and genital ulceration in conditions such as Behcet's disease: the use of neurophysiological studies to detect thalidomide neuropathy. Ann Rheum Dis, 1994; 53 828-832.

19 The Medicines (Exemption from Licences) (Special and Transitional Cases) Order 1971. HMSO, London 1971. 